\title{
NON-RESONANT FREDHOLM ALTERNATIVE AND ANTI-MAXIMUM PRINCIPLE FOR THE FRACTIONAL $p$-LAPLACIAN
}

\author{
LEANDRO M. DEL PEZZO AND ALEXANDER QUAAS
}

\begin{abstract}
In this paper we extend two nowadays classical results to a nonlinear Dirichlet problem to equations involving the fractional $p$-Laplacian. The first result is an existence in a non-resonant range more specific between the first and second eigenvalue of the fractional $p$-Laplacian. The second result is the anti-maximum principle for the fractional $p$-Laplacian.
\end{abstract}

\section{Dedicated to Paul H. Rabinowitz}

Mathematics Subject Classification (2010). 35R11, 47G20, 45G05.

Keywords. Fractional $p$-Laplacian, existence results, non-resonant, anti-maximum principle.

\section{INTRODUCTION}

This paper deals with existence and qualitative results for the following nonlinear Dirichlet problem with the fractional $p$-Laplacian

$$
\begin{cases}\left(-\Delta_{p}\right)^{s} u=\lambda|u|^{p-2} u+f(x) & \text { in } \Omega, \\ u=0 & \text { in } \Omega^{c}:=\mathbb{R}^{N} \backslash \Omega .\end{cases}
$$

Here and in the rest of this introduction, $\Omega$ is a smooth bounded open of $\mathbb{R}^{N}$, $s \in(0,1)$, and $p \in(1, \infty)$. The fractional $p$-Laplacian is a nonlocal version of the $p$-Laplacian and is an extension of the fractional Laplacian $(p=2)$. More precisely, the fractional $p$-Laplacian is define as

$$
\left(-\Delta_{p}\right)^{s} u(x)=2 \mathcal{K} \text { P.V. } \int_{\mathbb{R}^{N}} \frac{|u(x)-u(y)|^{p-2}(u(x)-u(y))}{|x-y|^{N+s p}} d y,
$$

with

$$
\mathcal{K}=p(1-s)\left(\int_{S^{N-1}}|\langle\omega, e\rangle|^{p} d \mathcal{H}^{N-1}(\omega)\right)^{-1}, \quad e \in S^{N-1} .
$$

where $S^{N-1}$ denotes the unit sphere in $\mathbb{R}^{N}$ and $\mathcal{H}^{N-1}$ denotes the $N$-1-dimensional Hausdorff measure. For more details, see [14, 17].

A pioneer work on existence of nonlinear one dimensional integral equation (with $L^{2}$ kernels) under non-resonant case can be found in [27]. Beside that let as recall that the Fredholm alternative fails for $p$-Laplacian and the situation is more much complex that in the linear case. This can be found in a large number of results around Fredholm type alternative for the $p$-Laplacian, see for instance [9, 23, 28, $29,30,31,43,50,51]$ and the references therein. 
For the fractional Laplacian, the standard Fredholm alternative for compact operator can be applied. Observe that the spectrum for the fractional Laplacian is studied in $[48,46]$.

Let star by describing our existence results. Denote by $\lambda_{1}(s, p)$ and $\lambda_{2}(s, p)$ the first and second eigenvalues respectively for the fractional $p$-Laplacian with Dirichlet boundary condition. See Section 2 for the definition and basic properties of the eigenvalues of the fractional $p$-Laplacian.

First, by standard minimization argument, we show that if $\lambda<\lambda_{1}(s, p)$, then there is a unique weak solution of (1.1), see Section 3. Then, also in Section 3, we show the existence of solution to (1.2) for $\lambda \in\left(\lambda_{1}(s, p), \lambda_{2}(s, p)\right)$ and $f \in W^{-s, p^{\prime}}(\Omega)$. This existence part relies in an homotopy deformation of the degree as in [5], see also $[4,13,6]$.

More precisely, we can prove the following Theorem.

Theorem 1.1. Let $f \in W^{-s, p^{\prime}}(\Omega)$. If $\lambda_{1}(s, p)<\lambda<\lambda_{2}(s, p)$ then there is a weak solution of (1.1).

Let as observe that Fredholm type alternative for fully non-linear operator can be found in [11, Section 5]. Notice that using the ideas of [11] and [21] a different homotopy (respect to $s$ ) can be use to prove the above Theorem. Beside that let also mention that from [21] other existence results can be proved using bifurcation from infinity for (1.1). This results can be found for the case of the $p$-Laplacian for example in [9].

Our second aim is to show an anti-maximum principle for the fractional $p$-Laplacian. This principle has shown to be a powerful tool when analyzing nonlinear elliptic problems, see $[8,18,36,12]$ and the references therein. For the $p$-Laplacian operator, the anti-maximum principle is proven in [32], see also [10, 35]. On the other hand, the link between bifurcation theory and anti-maximum principle was observed by the first time in [8] (see for instance [8, Theorem 27] for a improvement of the the anti-maximum principle for the $p$-Laplacian operator).

In Section 4, before proving our anti-maximum principle we show the following maximum principle.

Theorem 1.2. Let $f \in W^{-s, p^{\prime}}(\Omega)$ be such that $f \not \equiv 0$.

(1) If $f \geq 0$ and $\lambda<\lambda_{1}(s, p)$, then $u>0$ a.e. in $\Omega$ for any super-solution $u$ of $(1.1)$.

(2) If $f \leq 0$, and $\lambda<\lambda_{1}(s, p)$, then $u<0$ a.e. in $\Omega$ for any sub-solution $u$ of $(1.1)$.

Thus, we show the following anti-maximum principle.

Theorem 1.3. Let $f \in W^{-s, p^{\prime}}(\Omega)$ be such that $f \not \equiv 0$. Then there is $\delta=\delta(f)>0$ such that

(1) if $f \geq 0$ and $\lambda \in\left(\lambda_{1}(s, p), \lambda_{1}(s, p)+\delta\right)$ then any weak solution $u$ of $(1.1)$ satisfies $u<0$ a.e. in $\Omega$.

(2) if $f \leq 0$ and $\lambda \in\left(\lambda_{1}(s, p), \lambda_{1}(s, p)+\delta\right)$ then any weak solution $u$ of (1.1) satisfies $u>0$ a.e. in $\Omega$. 
Let's comment that, for the spectral fractional Laplacian (this is a different operator than $\left.(-\Delta)^{s}\right)$, the anti-maximum principle is only proved in the case $s=$ $1 / 2$, see [7]. In fact, we would like to mention that the proof in [7] can be easily extended to the case $s \in(0,1)$. See also [34] where the anti-maximum principle is shown for non-singular kernel. So, as far we know, Theorem 1.3 is new even for the case $p=2$. Therefore, we extent in particular the now classical anti-maximum principle of Clement and Peletier (see [18]) for all the range $s \in(0,1)$ and $p \in(1, \infty)$.

We want to observe that, our proof of the previous theorem is not a straightforward adaptation of the proof given in the local case due to we do not have a suitable Hopf's lemma for the fractional $p$-Laplacian. To overcome this problem we will use Picone's identity (see Lemma 2.9) and show a lower bound for the measures of the negative (positive) sets of the weak super(sub)-solutions of (1.1) (see Lemma 4.5 and Remark 4.6 below).

In the linear case $(\mathrm{p}=2)$, thanks to the regularity results up to the boundary and the Hopf lemma, we can prove a more general result improving Theorems 1.2 and 1.3 .

Theorem 1.4. Let $\Omega$ be a bounded domain with $C^{1,1}$ boundary, $w_{1}$ be a positive eigenfunction of $(-\Delta)^{s}$ associated to $\lambda_{1}(s, 2)$. For any $f \in L^{\infty}(\Omega)$ with $\int_{\Omega} f(x) w_{1} d x \neq$ 0 , there is $\delta=\delta(f)>0$ such that

(1) if $\int_{\Omega} f(x) w_{1} d x>0$ then any weak solution $u$ of (1.1) satisfies (a) $u<0$ in $\Omega$ if $\lambda \in\left(\lambda_{1}(s, 2), \lambda_{1}(s, 2)+\delta\right)$;

(b) $u>0$ in $\Omega$ if $\lambda \in\left(\lambda_{1}(s, 2)-\delta, \lambda_{1}(s, 2)\right)$;

(2) if $\int_{\Omega} f(x) w_{1} d x<0$ then any weak solution $u$ of (1.1) satisfies

(a) $u>0$ in $\Omega$ if $\lambda \in\left(\lambda_{1}(s, 2), \lambda_{1}(s, 2)+\delta\right)$;

(b) $u<0$ in $\Omega$ if $\lambda \in\left(\lambda_{1}(s, 2)-\delta, \lambda_{1}(s, 2)\right)$.

The paper is organize as follows. In Section 2 we review some preliminaries including the eigenvalue problems. In Section 3 we prove our existence results. Finally, in Section 4 we prove Theorems 1.2, 1.3 and 1.4.

\section{Preliminaries}

Let's start by introducing the notation and definitions that we will use in this work. We also gather some preliminaries properties which will be useful in the forthcoming sections.

Here and hereafter, $s \in(0,1), p \in(1, \infty)$ and we will denote by $\Omega$ an open set in $\mathbb{R}^{N}$. Given a subset $A$ of $\mathbb{R}^{N}$ we set $A^{c}=\mathbb{R}^{N} \backslash A$, and $A^{2}=A \times A$. For all function $u: \Omega \rightarrow \mathbb{R}$ we define

$$
\begin{gathered}
u_{+}(x):=\max \{u(x), 0\} \quad \text { and } \quad u_{-}(x):=\max \{-u(x), 0\}, \\
\Omega_{+}:=\{x \in \Omega: u(x)>0\} \quad \text { and } \quad \Omega_{-}:=\{x \in \Omega: u(x)<0\} .
\end{gathered}
$$




\subsection{Fractional Sobolev spaces.}

The fractional Sobolev spaces $W^{s, p}(\Omega)$ is defined to be the set of functions $u \in$ $L^{p}(\Omega)$ such that

$$
|u|_{W^{s, p}(\Omega)}^{p}:=\int_{\Omega^{2}} \frac{|u(x)-u(y)|^{p}}{|x-y|^{N+s p}} d x d y<\infty .
$$

The fractional Sobolev spaces admit the following norm

$$
\|u\|_{W^{s, p}(\Omega)}:=\left(\|u\|_{L^{p}(\Omega)}^{p}+|u|_{W^{s, p}(\Omega)}^{p}\right)^{\frac{1}{p}},
$$

where

$$
\|u\|_{L^{p}(\Omega)}^{p}:=\int_{\Omega}|u(x)|^{p} d x .
$$

The space $W^{s, p}\left(\mathbb{R}^{N}\right)$ is defined similarly.

We will denote by $\widetilde{W^{s, p}}(\Omega)$ the space of all $u \in W^{s, p}(\Omega)$ such that $\tilde{u} \in W^{s, p}\left(\mathbb{R}^{N}\right)$, where $\tilde{u}$ is the extension by zero of $u$. The dual space of $\widetilde{W}^{s, p}(\Omega)$ is denoted by $W^{-s, p^{\prime}}(\Omega)$ and the corresponding dual pairing is denoted by $\langle\cdot, \cdot\rangle$.

Remark 2.1. By [26, Lemma 6.1], if $\Omega$ is bounded then there is a suitable constant $C=C(N, s, p)>0$ such that for any $u \in \widetilde{W}^{s, p}(\Omega)$ we get

$$
\begin{aligned}
|u|_{W^{s, p}\left(\mathbb{R}^{N}\right)}^{p} & \geq \int_{\Omega \times \Omega^{c}} \frac{|u(x)|^{p}}{|x-y|^{N+s p}} d x d y=\int_{\Omega}|u(x)|^{p} \int_{\Omega^{c}} \frac{1}{|x-y|^{N+s p}} d y d x \\
& \geq \frac{C}{|\Omega|^{s p / N}}\|u\|_{L^{p}(\Omega)}^{p},
\end{aligned}
$$

where $|\Omega|$ denotes the Lebesgue measure of $\Omega$. Hence, the seminorm $|\cdot|_{W^{s, p}\left(\mathbb{R}^{N}\right)}$ is a norm in $\widetilde{W}^{s, p}(\Omega)$ equivalent to the standard norm.

If $\Omega$ is bounded, we set

$$
\widehat{W}^{s, p}(\Omega):=\left\{u \in L_{l o c}^{p}\left(\mathbb{R}^{N}\right): \exists U \supset \supset \Omega \text { s.t. } u \in W^{s, p}(U),[u]_{s, p}<\infty\right\},
$$

where

$$
[u]_{s, p}:=\int_{\mathbb{R}^{N}} \frac{|u(x)|^{p-1}}{(1+|x|)^{N+s p}} d x .
$$

Observe that $\widetilde{W}^{s, p}(\Omega) \subset \widehat{W}^{s, p}(\Omega)$.

We will denote by $p_{s}^{\star}$ the fractional critical Sobolev exponent, that is

$$
p_{s}^{\star}:= \begin{cases}\frac{N p}{N-s p} & \text { if } s p<N, \\ +\infty & \text { if } s p \geq N .\end{cases}
$$

Remark 2.2. If $\mathcal{X}=W^{s, p}(\Omega)$ or $\widetilde{W}^{s, p}(\Omega)$ or $\widehat{W}^{s, p}(\Omega)$ and $u \in \mathcal{X}$ then $u_{+}, u_{-} \in \mathcal{X}$ owing to

$$
\left|u_{-}(x)-u_{-}(y)\right| \leq|u(x)-u(y)| \quad \text { and } \quad\left|u_{+}(x)-u_{+}(y)\right| \leq|u(x)-u(y)|,
$$

for all $x, y \in \Omega$.

Further information on fractional Sobolev spaces and many references may be found in $[1,25,26,38,39]$. 


\subsection{Dirichlet Problems.}

Let $\Omega$ be a bounded open set in $\mathbb{R}^{N}, s \in(0,1)$, and $f \in W^{-s, p^{\prime}}(\Omega)$. We say that $f \geq(\leq) 0$ if for any $v \in \widetilde{W}^{s, p}(\Omega), v \geq 0$ we have that $\langle f, v\rangle \geq(\leq) 0$.

We say that $u \in \widehat{W}^{s, p}(\Omega)$ is a weak super-solution of

$$
\begin{cases}\left(-\Delta_{p}\right)^{s} u=f(x) & \text { in } \Omega, \\ u=0 & \text { in } \Omega^{c},\end{cases}
$$

if $u \geq 0$ a.e. in $\Omega^{c}$ and

$$
\mathcal{K} \int_{\mathbb{R}^{2 N}} \frac{|u(x)-u(y)|^{p-2}(u(x)-u(y))(v(x)-v(y))}{|x-y|^{N+s p}} d x d y \geq\langle f, v\rangle,
$$

for each $v \in \widetilde{W^{s}, p}(\Omega), v \geq 0$.

A function $u \in \widehat{W}^{s, p}(\Omega)$ is a weak sub-solution of $(2.1)$ if $-u$ is a weak supersolution. Finally, a function $u \in \widehat{W}^{s, p}(\Omega)$ is a weak solution of (2.1) if and only if is both a weak super-solution and a weak sub-solution.

Our next result is a minimum principle.

Lemma 2.3. Let $f \in W^{-s, p^{\prime}}(\Omega)$ be such that $f \geq 0$, and $u$ be a weak super-solution of (2.1). Then either $u>0$ a.e. in $\Omega$ or $u=0$ a.e. in $\Omega$.

Proof. Since $u$ is a weak super-solution of (2.1), it follows from the comparison principle (see [39, Proposition 2.10]) that $u \geq 0$ in $\mathbb{R}^{N}$. Moreover, if $\Omega$ is connected, by [15, Theorem A.1], we get if $u \neq 0$ a.e. in $\Omega$ then $u>0$ a.e. in $\Omega$.

Then, we only need to show that $u \not \equiv 0$ in $\Omega$ if only if $u \not \equiv 0$ in all connected components of $\Omega$. That is, we only need to show that if $u \not \equiv 0$ in $\Omega$ then $u \not \equiv 0$ in all connected components of $\Omega$.

Suppose, to the contrary, that is $u \not \equiv 0$ and there is a connected component $U$ of $\Omega$ such that $u \equiv 0$ in $U$. Moreover, for any nonnegative function $v \in \widetilde{W}^{s, p}(U)$ we get

$$
\begin{aligned}
0 & \leq \int_{\mathbb{R}^{2 N}} \frac{|u(x)-u(y)|^{p-2}(u(x)-u(y))(v(x)-v(y))}{|x-y|^{N+s p}} d x d y \\
& =-2 \int_{U} \int_{U^{c}} \frac{|u(x)|^{p-2} u(x) v(y)}{|x-y|^{N+s p}} d x d y
\end{aligned}
$$

due to $u \equiv 0$ in $U$. Then $u=0$ a.e. in $U^{c}$, that is $u=0$ a.e. in $\mathbb{R}^{N}$, which is a contradiction with the fact that $u \neq 0$ a.e. in $\Omega$.

To prove the Theorem 1.1, we will use the homotopy property of the LeraySchauder degree. For this reason, we need to recall some properties of the Dirichlet problem for the fractional $p$-Laplace equations.

Let $f \in W^{-s, p^{\prime}}(\Omega)$. If $\Omega$ is a smooth bounded domain, using the fractional Sobolev compact embedding theorem (see $[1,25]$ ), it is easily seen that $(2.1)$ has a unique weak solution $u_{f} \in \widetilde{W}^{s, p}(\Omega)$. Moreover, the operator

$$
\begin{aligned}
\mathcal{R}_{s, p}: W^{-s, p^{\prime}}(\Omega) & \rightarrow \widetilde{W}^{s, p}(\Omega) \\
f & \rightarrow u_{f}
\end{aligned}
$$

is continuous, see [21] 
Now, let $\Omega$ be a smooth bounded domain, $f \in W^{-s, p^{\prime}}(\Omega)$ and $t \in \mathbb{R}$, we define the operator $T_{t}: \widetilde{W}^{s, p}(\Omega) \rightarrow \widetilde{W}^{s, p}(\Omega)$ by

$$
T_{t}(u):=\mathcal{R}_{s, p}\left(\lambda|u|^{p-2} u+t f\right) .
$$

Notice that by the fractional Sobolev compact embedding theorem and the continuity of $\mathcal{R}_{s, p}$ we have that $T_{t}$ is a completely continuous operator.

\subsection{Eigenvalue Problems.}

Now we study the following eigenvalue problems

$$
\begin{cases}\left(-\Delta_{p}\right)^{s} u=\lambda|u|^{p-2} u & \text { in } \Omega, \\ u=0 & \text { in } \Omega^{c},\end{cases}
$$

We say that $\lambda$ is an eigenvalue of $\left(-\Delta_{p}\right)^{s}$ if there is a function $u \in \widetilde{W}^{s, p}(\Omega) \backslash\{0\}$ such that for any $v \in \widetilde{W}^{s, p}(\Omega)$

$$
\mathcal{K} \int_{\mathbb{R}^{2 N}} \frac{|u(x)-u(y)|^{p-2}(u(x)-u(y))(v(x)-v(y))}{|x-y|^{N+s p}} d x d y=\lambda \int_{\Omega}|u|^{p-2} u v d x .
$$

The function $u$ is a corresponding eigenfunction of $\left(-\Delta_{p}\right)^{s}$ associated to $\lambda$.

Before showing the existence of a sequence of eigenvalues, we need to introduce some additional notation. Following [17], we define

$$
\mathcal{S}^{s, p}:=\left\{u \in \widetilde{W}^{s, p}(\Omega):\|u\|_{L^{p}(\Omega)}=1\right\}
$$

and

$$
\mathcal{W}_{m}^{s, p}:=\left\{K \subset \mathcal{S}^{s, p}: K \text { is symmetric and compact, } i(K) \geq m\right\}
$$

for $m \in \mathbb{N}$. Here $i$ denotes the Krasnosel'skiĩ genus.

For the proof of the following theorem see $[16,17,33,21,40]$ (for the local case, see $[19,44,3,41,42])$.

Theorem 2.4. Let $\Omega$ a smooth bounded domain of $\mathbb{R}^{N}$. Then there is a sequence of eigenvalues of $\left(-\Delta_{p}\right)^{s}$

$$
\lambda_{m}(s, p)=\inf _{K \in \mathcal{W}_{m}^{s, p}} \max _{u \in K} \mathcal{K}|u|_{W^{s, p}\left(\mathbb{R}^{N}\right)} .
$$

Moreover

- If $u$ is an eigenfunction of $\left(-\Delta_{p}\right)^{s}$ then $u \in L^{\infty}(\Omega)$.

- $\lambda_{1}(s, p)$ is the first eigenvalue of $\left(-\Delta_{p}\right)^{s}$, that is

$$
\lambda_{1}(s, p)=\inf \left\{\mathcal{K}|u|_{W^{s, p}\left(\mathbb{R}^{N}\right)}^{p}: u \in \mathcal{S}^{s, p}\right\} .
$$

- $\lambda_{1}(s, p)$ is simple and isolated.

- Any eigenfunction of $\left(-\Delta_{p}\right)^{s}$ associated to $\lambda_{1}(s, p)$ have constant sign.

- If $u$ is an eigenfunction of $\left(-\Delta_{p}\right)^{s}$ associated to $\lambda>\lambda_{1}(s, p)$ then $u$ must be sign-changing. 
- $\lambda_{2}(s, p)$ is the second eigenvalue

$$
\begin{aligned}
\lambda_{2}(s, p) & =\inf _{\gamma \in \Gamma\left(w_{1},-w_{1}\right)} \max _{u \in \operatorname{Im} \gamma(0,1)} \mathcal{K}|u|_{W^{s, p}\left(\mathbb{R}^{N}\right)}^{p} \\
& =\inf \left\{\lambda: \lambda>\lambda_{1}(s, p) \text { is an eigenvalue of }\left(-\Delta_{p}\right)^{s}\right\},
\end{aligned}
$$

where $w_{1}$ is an eigenfunction of $\left(-\Delta_{p}\right)^{s}$ associated to $\lambda_{1}(s, p)$ and $\Gamma\left(w_{1},-w_{1}\right)$ is the set of continuous paths on $\mathcal{S}^{s, p}$ connecting to $w_{1}$ and $-w_{1}$.

Remark 2.5. It is not difficult to see that, if $u \in \widetilde{W}^{s, p}(\Omega)$ is such that

$$
\lambda_{1}(s, p)=\frac{\mathcal{K}|u|_{W^{s, p}\left(\mathbb{R}^{N}\right)}^{p}}{\|u\|_{L^{p}(\Omega)}^{p}}
$$

then $u$ is eigenfunction of $\left(-\Delta_{p}\right)^{s}$ associated to $\lambda_{1}(s, p)$.

Let finally observe that in [21], we also prove that $\lambda_{1}(\cdot, p)$ is continuous.

\subsection{Regularity results.}

Here, we study the regularity up to the boundary of weak solutions of (1.1) when $f \in L^{\infty}(\Omega)$. For this, we need the following results

Lemma 2.6. Let $f \in L^{\infty}(\Omega)$ and $\lambda \in \mathbb{R}$. If $u$ is a weak solution of (1.1) then $u \in L^{\infty}(\Omega)$.

Proof. In this proof, we borrow ideas from [33, 47].

If $p s>N$, then $u \in L^{\infty}(\Omega)$ due to the fractional Sobolev embedding theorem. For the rest of the proof, we assume $s p \leq N$.

Let $u$ be a a weak solution of (1.1). Up to multiplying $u$ by a small constant we may assume that

where $\delta>0$ will be selected below.

$$
\|u\|_{L^{p}(\Omega)}=\sqrt{\delta}
$$

For any $k \in \mathbb{N}$, we define $v_{k}:=\left(u-1+2^{-k}\right)_{+}$and $U_{k}=\left\|v_{k}\right\|_{L^{p}(\Omega)}^{p}$. Observe that, for any $k \in \mathbb{N}$ we have that

$$
\begin{aligned}
& v_{k} \in \widetilde{W}^{s, p}(\Omega), \quad v_{k+1} \leq v_{k} \text { a.e. in } \mathbb{R}^{N} \text { and } \\
& \left\{x \in \Omega: v_{k+1}>0\right\} \subset\left\{x \in \Omega: v_{k}>2^{-(k+1)}\right\} .
\end{aligned}
$$

Moreover $U_{k} \rightarrow\left\|(u-1)_{+}\right\|_{L^{p}(\Omega)}$ as $k \rightarrow \infty$. Then, for any $k \in \mathbb{N}$

$$
\begin{aligned}
\mathcal{K}\left|v_{k}\right|_{W^{s, p}(\Omega)}^{p} & =\mathcal{K} \int_{\Omega^{2}} \frac{\left|v_{k+1}(x)-v_{k+1}(y)\right|^{p}}{|x-y|^{N+s p}} d x d y \\
& \leq \mathcal{K} \int_{\mathbb{R}^{2 N}} \frac{|u(x)-u(y)|^{p-2}(u(x)-u(y))\left(v_{k+1}(x)-v_{k+1}(y)\right)}{|x-y|^{N+s p}} d x d y \\
& =\lambda \int_{\Omega} v_{k+1}^{p} d x+\int_{\Omega} f(x) v_{k+1} d x \\
& \leq|\lambda| U_{k}+\|f\|_{L^{\infty}(\Omega)} \int_{\Omega} v_{k+1} d x \\
& \leq|\lambda| U_{k}+\|f\|_{L^{\infty}(\Omega)}\left|\left\{x \in \Omega: v_{k+1}>0\right\}\right|^{1-1 / p} U_{k}^{1 / p} .
\end{aligned}
$$

By (2.3), we get

$$
U_{k}=\left\|v_{k}\right\|_{L^{p}(\Omega)}^{p} \geq 2^{-p(k+1)}\left|\left\{x \in \Omega: v_{k+1}>0\right\}\right|
$$


then

$$
\mathcal{K}\left|v_{k}\right|_{W^{s, p}(\Omega)}^{p} \leq\left(|\lambda|+\|f\|_{L^{\infty}(\Omega)} 2^{(p-1)}\right) 2^{(p-1) k} U_{k} .
$$

Thus, given $q \in\left(p, p_{s}^{\star}\right)$, by the Holder inequality, the fractional Sobolev embedding theorem, (2.4) and (2.5), we have that

$$
\begin{aligned}
U_{k+1} & \leq\left\|v_{k+1}\right\|_{L^{q}(\Omega)}^{p}\left|\left\{x \in \Omega: v_{k+1}>0\right\}\right|^{1-p / q} \\
& \leq C\left|v_{k}\right|_{W^{s, p}(\Omega)}^{p}\left(2^{p(k+1)} U_{k}\right)^{1-p / q} \\
& \leq C\left(|\lambda|+\|f\|_{L^{\infty}(\Omega)} 2^{(p-1)}\right) 2^{\left(p-p^{2} / q\right)} 2^{\left(2 p-1-p^{2} / q\right) k} U_{k}^{2-q / p} \\
& \leq\left\{\left[1+C\left(|\lambda|+\|f\|_{L^{\infty}(\Omega)} 2^{(p-1)}\right) 2^{\left(p-p^{2} / q\right)}\right] 2^{\left(2 p-1-p^{2} / q\right)}\right\}^{k} U_{k}^{2-p / q} \\
& =C^{k} U_{k}^{\rho}
\end{aligned}
$$

where $C>1$ and $\rho=2-p / q>1$.

Now, we choose the number $\delta>0$ sufficiently small that

$$
\delta^{\rho}<\frac{1}{C^{1 /(\rho-1)}}
$$

and proceeding as in the end of the proof of [49, Proposition 7], we can conclude that $u \leq 1$ a.e. in $\Omega$. By replacing $u$ with $-u$ we obtain $\|u\|_{L^{\infty}(\Omega)} \leq 1$.

Then, by the previous lemma, [39, Theorem 1.1.] and [45, Proposition 1.1 and Theorem 1.2], we have

Theorem 2.7. Let $\Omega$ be a bounded domain with $C^{1,1}$ boundary, $f \in L^{\infty}(\Omega), \lambda \in \mathbb{R}$, and $\delta(x)=\operatorname{dist}(x, \partial \Omega)$. Then, there is $\alpha \in(0, s]$ and $C$, depending on $\Omega$ such that for all weak solution $u$ of (1.1), $u \in C^{\alpha}(\bar{\Omega})$ and

$$
\|u\|_{C^{\alpha}(\bar{\Omega})} \leq C\left(|\lambda|\|u\|_{L^{\infty}(\Omega)}+\|f\|_{L^{\infty}(\Omega)}\right) .
$$

In additional, if $p=2$ then $\alpha=s$ and

$$
u / \delta^{s} \in C^{\beta}(\bar{\Omega}) \quad \text { and } \quad\left\|u / \delta^{s}\right\|_{C^{\beta}(\bar{\Omega})} \leq D\left(|\lambda|\|u\|_{L^{\infty}(\Omega)}+\|f\|_{L^{\infty}(\Omega)}\right)
$$

where $\beta \in(0, \min \{s, 1-s\})$. The constants $\beta$ and $D$ depend only on $\Omega$ and $s$.

Finally, in the linear case, as a consequence of the fractional Hopf lemma (See $[37,22])$, we have the next result.

Lemma 2.8. Let $\Omega$ be a bounded domain with $C^{1,1}$ boundary, $\delta(x)=\operatorname{dist}(x, \partial \Omega)$, and $w_{1}$ be an eigenfunction of $(-\Delta)^{s}$. If $\left\{v_{n}\right\}_{n \in \mathbb{N}} \subset C^{s}(\bar{\Omega})$ is such that $v_{n} / \delta \in C(\bar{\Omega})$ and

$$
v_{n} \rightarrow w_{1} \quad \text { and } \quad \frac{v_{n}}{\delta^{s}} \rightarrow \frac{w_{1}}{\delta^{s}}
$$

strongly in $\bar{\Omega}$, then there is $n_{0} \in \mathbb{N}$ such that $v_{n}>0$ for all $n \geq n_{0}$.

\subsection{Picone inequality.}

For the proof of the following Picone inequality, see [2, Lemma 6.2 ].

Lemma 2.9. For every $a_{1}, a_{2} \geq 0$ and $b_{1}, b_{2}>0$

$$
\left|a_{1}-a_{2}\right|^{p} \geq\left|b_{1}-b_{2}\right|^{p-2}\left(b_{1}-b_{2}\right)\left(\frac{a_{1}^{p}}{b_{1}^{p-1}}-\frac{a_{2}^{p}}{b_{2}^{p-1}}\right) .
$$


The equality holds if and only if $\left(a_{1}, a_{2}\right)=k\left(b_{1}, b_{2}\right)$ for some constant $k$.

\section{Non-Resonant Fredholm alternative Problem}

Let's start this section proving the following existence results for equation (1.1) with $\lambda<\lambda_{1}(s, p)$. One of the principal results, that we will use through the rest of this work, is the fractional Sobolev compact embedding theorem. For this reason, throughout the rest of this work $\Omega$ is a smooth bounded domain of $\mathbb{R}^{N}$.

Theorem 3.1. Let $f \in W^{-s, p^{\prime}}(\Omega)$. If $\lambda<\lambda_{1}(s, p)$ then there is a weak solution of (1.1).

Proof. The proof of this theorem is standard. First observe that weak solutions of (1.1) are critical points of the functional $J: \widetilde{W}^{s, p}(\Omega) \rightarrow \mathbb{R}$, where

$$
J(u):=\frac{\mathcal{K}}{p}|u|_{W^{s, p}\left(\mathbb{R}^{N}\right)}^{p}-\frac{\lambda}{p}\|u\|_{L^{p}(\Omega)}^{p}-\langle f, u\rangle .
$$

It follows from $\lambda<\lambda_{1}(s, p)$ that $J$ is bounded below, coercive, strictly convex and sequentially weakly lower semi continuous. Thus $J$ has a unique critical point which is a global minimum.

Our next aim is to prove Theorem 1.1, to this end we will use the homotopy property of the Leray-Schauder degree. We first prove an a priori bound for the fixed points of $T_{t}$.

Lemma 3.2. If $\lambda_{1}(s, p)<\lambda<\lambda_{2}(s, p)$ then there exists $R>0$ such that for all $t \in[0,1]$ there is no solution of $\left(I-T_{t}\right) u=0$ for $|u|_{W^{s, p}\left(\mathbb{R}^{N}\right)} \geq R$

Proof. Suppose, to the contrary, that is for all $n \in \mathbb{N}$ there exist $t_{n} \in[0,1]$ and $u_{n} \in \widetilde{W}^{s, p}(\Omega)$ such that $\left(I-T_{t_{n}}\right) u_{n}=0$ and $\left|u_{n}\right|_{W^{s, p}\left(\mathbb{R}^{N}\right)} \rightarrow \infty$ as $n \rightarrow \infty$. Let define

$$
v_{n}=\frac{u_{n}}{\left|u_{n}\right|_{W^{s, p}\left(\mathbb{R}^{N}\right)}} \quad \forall n \in \mathbb{N} .
$$

Then for all $n \in \mathbb{N}$, we have that $v_{n}$ is a weak solution of

$$
\begin{cases}\left(-\Delta_{p}\right)^{s} u=\lambda_{n}|u|^{p-2} u+\frac{t_{n} f(x)}{\left|u_{n}\right|_{W^{s, p}\left(\mathbb{R}^{N}\right)}^{p-1}} & \text { in } \Omega, \\ u=0 & \text { in } \Omega^{c} .\end{cases}
$$

Using the fractional Sobolev compact embedding theorem, up to a subsequence (still denoted by $v_{n}$ )

$$
\begin{array}{ll}
v_{n} \rightarrow v & \text { weakly in } \widetilde{W}^{s, p}(\Omega), \\
v_{n} \rightarrow v & \text { strongly in } L^{p}(\Omega) .
\end{array}
$$

Thus, $|v|_{W^{s, p}\left(\mathbb{R}^{N}\right)}=1$ and since $t_{n} f /\left|u_{n}\right|_{W^{s, p}\left(\mathbb{R}^{N}\right)}^{p-1} \rightarrow 0$ strongly in $W^{-s, p^{\prime}}(\Omega)$, we have that $v$ is a weak solution of (1.1) with $f=0$ getting a contradiction since $\lambda_{1}(s, p)<\lambda<\lambda_{2}(s, p)$.

Now we are in position to proof Theorem 1.1. 
Proof of Theorem 1.1. By Lemma 3.2, the Leray-Schauder degree $d\left(I-T_{t}, B(0, R), 0\right)$ is well define and constant for all in $t \in[0,1]$ by the invariance of the degree by homotopy. Thus $d\left(I-T_{t}, B(0, R), 0\right)=-1$ since $d\left(I-T_{0}, B(0, R), 0\right)=-1$ by Theorem 5.3 of [21], from here the existence result follows.

Observe that, in the above proof, the fact $d\left(I-T_{0}, B(0, R), 0\right) \neq 0$ can be established without using the results of [21] as a consequence of Borsuk theorem (see for example [24, Theorem 8.3]).

\section{Maximum And ANTI-MAXimum PRINCIPLE}

In this section, we will denote by $w_{1}$ the positive eigenfunction of $\left(-\Delta_{p}\right)^{s}$ associated to $\lambda_{1}(s, p)$ whose $L^{p}$-norm is equal to 1 . Since $w_{1} \in L^{\infty}(\Omega)$, by [39], there is $\alpha \in(0,1)$ such that $w_{1} \in C^{\alpha}(\bar{\Omega})$.

We start proving Theorem 1.2.

Proof of Theorem 1.2. We only prove the first statement; the another statement can be proved in an analogous way.

Since $u \geq 0$ a.e. in $\Omega^{c}$ we have that $u_{-} \in \widetilde{W}^{s, p}(\Omega)$. Then

$$
\begin{aligned}
& \mathcal{K} \int_{\mathbb{R}^{2 N}} \frac{|u(x)-u(y)|^{p-2}(u(x)-u(y))\left(u_{-}(x)-u_{-}(y)\right)}{|x-y|^{N+s p}} d x d y= \\
&=-\lambda \int_{\Omega}\left|u_{-}\right|^{p} d x+\left\langle f, u_{-}\right\rangle,
\end{aligned}
$$

consequently

$$
\begin{aligned}
& \lambda \int_{\Omega}\left|u_{-}\right|^{p} d x= \\
& =-\mathcal{K} \int_{\mathbb{R}^{2 N}} \frac{|u(x)-u(y)|^{p-2}(u(x)-u(y))\left(u_{-}(x)-u_{-}(y)\right)}{|x-y|^{N+s p}} d x d y+\left\langle f, u_{-}\right\rangle \\
& \geq \mathcal{K} \int_{\mathbb{R}^{2 N}} \frac{\left|u_{-}(x)-u_{-}(y)\right|^{p}}{|x-y|^{N+s p}} d x d y .
\end{aligned}
$$

Thus, if $u_{-} \not \equiv 0$ then

$$
\lambda \geq \mathcal{K} \frac{\int_{\mathbb{R}^{2 N}} \frac{\left|u_{-}(x)-u_{-}(y)\right|^{p}}{|x-y|^{N+s p}} d x d y}{\int_{\Omega}\left|u_{-}\right|^{p} d x} \geq \lambda_{1}(s, p),
$$

a contradiction. Therefore $u \geq 0$ in $\mathbb{R}^{N}$. Moreover, proceeding as in the proof of Lemma 2.3, we have that $u \not \equiv 0$ in all connected components of $\Omega$. Finally, by [20, Theorem 2.9], $u>0$ a.e. in $\Omega$.

Before proving Theorem 1.3, we show some previous results.

Lemma 4.1. Let $\lambda \geq \lambda_{1}(s, p)$, and $f \in W^{-s, p^{\prime}}(\Omega)$ be such that $f \geq 0$ and $f \not \equiv 0$. Then the problem (1.1) has no non-negative weak super-solutions. 
Proof. Suppose, to the contrary, there is a non-negative weak super-solution $u$ of (1.1). Then, by Lemma 2.3,u>0 a.e. in $\Omega$. By the definition of $\widehat{W}^{s, p}(\Omega)$, let $U \supset \supset \Omega$ be such that

$$
\|u\|_{W^{s, p}(U)}+\int_{\mathbb{R}^{N}} \frac{|u|^{p-1}}{(1+|x|)^{N+s p}} d x<\infty
$$

$n \in \mathbb{N}$ and $u_{n}:=u+\frac{1}{n}$.

We begin by proving that $v_{n}:=\frac{w_{1}^{p}}{u_{n}^{p-1}} \in \widetilde{W}^{s, p}(\Omega)$. It is immediate that $v_{n}>0$ in $\Omega, v_{n}=0$ in $\Omega^{c}$, and since $w_{1} \in L^{\infty}(\Omega)$ we have that $v_{n} \in L^{p}(\Omega)$.

On the other hand

$$
\begin{aligned}
\left|v_{n}(x)-v_{n}(y)\right|= & \left|\frac{w_{1}(x)^{p}-w_{1}(y)^{p}}{u_{n}(x)^{p-1}}+\frac{w_{1}(y)^{p}\left(u_{n}(y)^{p-1}-u_{n}(x)^{p-1}\right)}{u_{n}(y)^{p-1} u_{n}(x)^{p-1}}\right| \\
\leq & n^{p-1}\left|w_{1}(x)^{p}-w_{1}(y)^{p}\right|+\left\|w_{1}\right\|_{L^{\infty}(\Omega)}^{p} \frac{\left|u_{n}(x)^{p-1}-u_{n}(y)^{p-1}\right|}{u_{n}(y)^{p-1} u_{n}(x)^{p-1}} \\
\leq & n^{p-1} p\left(w_{1}(x)^{p-1}+w_{1}(y)^{p-1}\right)\left|w_{1}(x)-w_{1}(y)\right| \\
& +\left\|w_{1}\right\|_{L^{\infty}(\Omega)}^{p}(p-1) \frac{\left|u_{n}(x)^{p-2}+u_{n}(y)^{p-2}\right|}{u_{n}(y)^{p-1} u_{n}(x)^{p-1}}\left|u_{n}(x)-u_{n}(y)\right| \\
\leq & 2\left\|w_{1}\right\|_{L^{\infty}(\Omega)}^{p-1} n^{p-1} p\left|w_{1}(x)-w_{1}(y)\right| \\
& +n\left\|w_{1}\right\|_{L^{\infty}(\Omega)}^{p}(p-1)\left(\frac{1}{u_{n}(y)}+\frac{1}{u_{n}(x)}\right)|u(x)-u(y)| \\
\leq & C\left(n, p,\left\|w_{1}\right\|_{L^{\infty}(\Omega)}\right)\left(\left|w_{1}(x)-w_{1}(y)\right|+|u(x)-u(y)|\right),
\end{aligned}
$$

for all $(x, y) \in \mathbb{R}^{N} \times \mathbb{R}^{N}$. Hence $v_{n} \in W^{s, p}(U)$ for all $m \in \mathbb{N}$ due to $w_{1}, u \in W^{s, p}(U)$. Then, since $v_{n}=0$ in $\Omega^{c}$, and $v_{n} \in W^{s, p}(U)$ with $\Omega \subset \subset U$, we have

$$
\begin{aligned}
& \int_{\mathbb{R}^{2 N}} \frac{\left|v_{n}(x)-v_{n}(y)\right|^{p}}{|x-y|^{N+s p}} d x d y= \\
& =\int_{U^{2}} \frac{\left|v_{n}(x)-v_{n}(y)\right|^{p}}{|x-y|^{N+s p}} d x d y+2 \int_{U \times U^{c}} \frac{\left|v_{n}(x)\right|^{p}}{|x-y|^{N+s p}} d x d y \\
& =\int_{U^{2}} \frac{\left|v_{n}(x)-v_{n}(y)\right|^{p}}{|x-y|^{N+s p}} d x d y+2 \int_{\Omega \times U^{c}} \frac{\left|v_{n}(x)\right|^{p}}{|x-y|^{N+s p}} d x d y \\
& =\int_{U^{2}} \frac{\left|v_{n}(x)-v_{n}(y)\right|^{p}}{|x-y|^{N+s p}} d x d y+2 n^{p}\left\|w_{1}\right\|_{L^{\infty}(\Omega)} \int_{\Omega \times U^{c}} \frac{d x d y}{|x-y|^{N+s p}} \\
& <\infty \text {, }
\end{aligned}
$$

that is $v_{n} \in W^{s, p}\left(\mathbb{R}^{N}\right)$. Therefore, $v_{n} \in \widetilde{W}^{s, p}(\Omega)$.

Now, set

$$
\begin{aligned}
& L\left(w_{1}, u_{n}\right):= \\
& \quad\left|w_{1}(x)-w_{1}(y)\right|^{p}-\left|u_{n}(x)-u_{n}(y)\right|^{p-2}\left(u_{n}(x)-u_{n}(y)\right)\left(\frac{w_{1}(x)^{p}}{u_{n}(x)^{p-1}}-\frac{w_{1}(x)^{p}}{u_{n}(y)^{p-1}}\right)
\end{aligned}
$$

By Lemma 2.9, we have 


$$
\begin{aligned}
0 & \leq \mathcal{K} \int_{\Omega^{2}} \frac{L\left(w_{1}, u_{n}\right)(x, y)}{|x-y|^{N+s p}} d x d y \leq \mathcal{K} \int_{\mathbb{R}^{2 N}} \frac{L\left(w_{1}, u_{n}\right)(x, y)}{|x-y|^{N+s p}} d x d y \\
& \leq \mathcal{K} \int_{\mathbb{R}^{2 N}} \frac{\left|w_{1}(x)-w_{1}(y)\right|^{p}}{|x-y|^{N+s p}} d x d y \\
& \quad-\mathcal{K} \int_{\mathbb{R}^{2 N}} \frac{|u(x)-u(y)|^{p-2}(u(x)-u(y))}{|x-y|^{N+s p}}\left(v_{n}(x)-v_{n}(y)\right) d x d y \\
& \leq \lambda_{1}(s, p) \int_{\Omega} w_{1}(x)^{p} d x-\lambda \int_{\Omega} u(x)^{p-1} v_{n}(x) d x-\left\langle f, v_{n}\right\rangle \\
& \leq \lambda_{1}(s, p) \int_{\Omega} w_{1}(x)^{p} d x-\lambda \int_{\Omega} u(x)^{p-1} \frac{w_{1}(x)^{p}}{u_{n}(x)^{p-1}} d x-\left\langle f, \frac{w_{1}^{p}}{u_{n}^{p-1}}\right\rangle \\
& \leq \lambda_{1}(s, p) \int_{\Omega} w_{1}(x)^{p} d x-\lambda \int_{\Omega} u(x)^{p-1} \frac{w_{1}(x)^{p}}{u_{n}(x)^{p-1}} d x,
\end{aligned}
$$

due to $w_{1}$ is the positive eigenvalue associated to $\lambda_{1}(s, p), u \in \widehat{W}^{s, p}(\Omega)$ is a weak super-solution of (1.1) and $f \geq 0$.

Since $\lambda_{1}(s, p) \leq \lambda$, by the Fatou's lemma and the dominated convergence theorem

$$
\int_{\Omega^{2}} \frac{L\left(w_{1}, u\right)(x, y)}{|x-y|^{N+s p}} d x d y=0
$$

Then, again by Lemma $2.9, L\left(w_{1}, u\right)(x, y)=0$ a.e. in $\Omega$. and $u=k w_{1}$ a.e. in $\Omega$ for some constant $k>0$. Then

$$
\begin{aligned}
& \lambda_{1}(s, p) \int_{\Omega} u(x)^{p-1} \varphi(x) d x= \\
&= \mathcal{K} \int_{\mathbb{R}^{2}} \frac{|u(x)-u(y)|^{p-2}(u(x)-u(y))(\varphi(x)-\varphi(y))}{|x-y|^{N+s p}} d x d y \\
& \geq \lambda \int_{\Omega} u(x)^{p-1} \varphi(x) d x+\langle f, \varphi\rangle,
\end{aligned}
$$

for any $\varphi \in \widetilde{W}^{s, p}(\Omega), \varphi \geq 0$. This is a contradiction since $\lambda \geq \lambda_{1}(s, p)$ and $f \geq 0$, $f \not \equiv 0$.

Remark 4.2. Observe that, Lemma 4.1 implies that if $\lambda \geq \lambda_{1}(s, p)$, and $f \in$ $W^{-s, p^{\prime}}(\Omega)$ is such that $f \leq 0$ and $f \neq \equiv 0$, then the problem (1.1) has no nonpositive weak sub-solutions.

Corollary 4.3. Let $f \in W^{-s, p^{\prime}}(\Omega)$ be such that $f \geq 0$ and $f \not \equiv 0$. Then the problem (1.1) with $\lambda=\lambda_{1}(s, p)$ has no weak super-solutions.

Proof. We argue by contradiction. If there would exists a weak super-solution $u$ of (1.1) with $\lambda=\lambda_{1}(s, p)$. By Lemma $4.1, u_{-} \not \equiv 0$ in $\Omega$. Since $u_{-} \in \widetilde{W}^{s, p}(\Omega)$ we get, 
by the characterization of $\lambda_{1}(s, p)$ given in Theorem 2.4,

$$
\begin{aligned}
& -\lambda_{1}(s, p) \int_{\Omega} u_{-}(x)^{p} d x \leq \lambda_{1}(s, p) \int_{\Omega}|u(x)|^{p-2} u(x) u_{-}(x) d x+\left\langle f, u_{-}\right\rangle \\
& \leq \mathcal{K} \int_{\mathbb{R}^{2 N}} \frac{|u(x)-u(y)|^{p-2}\left(u(x)-u(y)\left(u_{-}(x)-u_{-}(y)\right)\right.}{|x-y|^{N+s p}} d x d y \\
& \leq-\mathcal{K} \int_{\Omega_{-}^{2}} \frac{\left|u_{-}(x)-u_{-}(y)\right|^{p}}{|x-y|^{N+s p}} d x d y-2 \mathcal{K} \int_{\Omega_{-} \times \Omega_{-}^{c}} \frac{\left(u_{-}(x)+u_{+}(y)\right)^{p-1} u_{-}(x)}{|x-y|^{N+s p}} d x d y \\
& \leq-\mathcal{K} \int_{\mathbb{R}^{2 N}} \frac{\left|u_{-}(x)-u_{-}(y)\right|^{p}}{|x-y|^{N+s p}} d x d y .
\end{aligned}
$$

Therefore

$$
\lambda_{1}(s, p) \geq \mathcal{K} \frac{\int_{\mathbb{R}^{2 N}} \frac{\left|u_{-}(x)-u_{-}(y)\right|^{p}}{|x-y|^{N+s p}} d x d y}{\int_{\Omega} u_{-}(x)^{p} d x},
$$

that is $u_{-}$is a corresponding eigenfunction to $\lambda_{1}(s, p)$ (see Remark 2.5). Then there is $k>0$ such that $u_{-}=k w_{1}$, and therefore $u_{-}>0$ in $\Omega$, that is $u<0$ in $\Omega$. Moreover

$$
\begin{aligned}
\lambda_{1}(s, p) & \int_{\Omega}|u(x)|^{p-2} u v d x \\
& =-\mathcal{K} \int_{\mathbb{R}^{2 N}} \frac{\left|u_{-}(x)-u_{-}(y)\right|^{p-2}\left(u_{-}(x)-u_{-}(y)\right)(v(x)-v(y))}{|x-y|^{N+s p}} d x d y \\
& \geq \mathcal{K} \int_{\mathbb{R}^{2 N}} \frac{|u(x)-u(y)|^{p-2}(u(x)-u(y))(v(x)-v(y))}{|x-y|^{N+s p}} d x d y \\
& \geq \lambda_{1}(s, p) \int_{\Omega}|u(x)|^{p-2} u v d x+\langle f, v\rangle
\end{aligned}
$$

for any $v \in \widetilde{W}^{s, p}(\Omega), v \geq 0$. This is a contradiction since $f \geq 0$, and $f \not \equiv 0$.

Remark 4.4. Note that, it follows straightforward from Corollary 4.3 that if $f \in$ $W^{-s, p^{\prime}}(\Omega)$ is such that $f \leq 0$ and $f \not \equiv 0$. Then the problem (1.1) with $\lambda=\lambda_{1}(s, p)$ has no weak sub-solutions.

Lemma 4.5. Let $\lambda \geq \lambda_{1}(s, p)$, and $f \in W^{-s, p^{\prime}}(\Omega)$ be such that $f \geq 0$ and $f \not \equiv 0$. Then there exist $\alpha>1$ and a constant $C>0$ such that for all $u$ is a weak supersolution of (1.1) we have that

$$
\left(\frac{C}{\lambda}\right)^{\alpha} \leq\left|\Omega_{-}\right|
$$

where $\Omega_{-}=\{x \in \Omega: u(x)<0\}$.

Proof. Let $u$ be a weak super-solution of (1.1). By Lemma 4.1, $u_{-} \not \equiv 0$ in $\Omega$. Taking $u_{-}$as test function, we have that

$$
\begin{aligned}
\mathcal{K} \int_{\mathbb{R}^{2 N}} \frac{|u(x)-u(y)|^{p-2}(u(x)-u(y))\left(u_{-}(x)-u_{-}(y)\right)}{|x-y|^{N+s p}} & d x d y \\
& \geq-\lambda \int_{\Omega}\left|u_{-}\right|^{p} d x+\left\langle f, u_{-}\right\rangle .
\end{aligned}
$$


If $p<q<p_{s}^{\star}$, by fractional Sobolev embedding theorem, then there is a constant $C$ such that

$$
\begin{aligned}
C \mathcal{K}\left\|u_{-}\right\|_{L^{q}(\Omega)}^{p} \leq \mathcal{K}\left|u_{-}\right|_{W^{s, p}\left(\mathbb{R}^{N}\right)}^{p} \\
\quad \leq-\mathcal{K} \int_{\mathbb{R}^{2 N}} \frac{|u(x)-u(y)|^{p-2}(u(x)-u(y))\left(u_{-}(x)-u_{-}(y)\right)}{|x-y|^{N+s p}} d x d y \\
\quad \leq-\mathcal{K} \int_{\mathbb{R}^{2 N}} \frac{|u(x)-u(y)|^{p-2}(u(x)-u(y))\left(u_{-}(x)-u_{-}(y)\right)}{|x-y|^{N+s p}} d x d y+\left\langle f, u_{-}\right\rangle \\
\quad \leq \lambda \int_{\Omega}\left|u_{-}\right|^{p} d x,
\end{aligned}
$$

and using the Hölder inequality

$$
C \mathcal{K}\left\|u_{-}\right\|_{L^{q}(\Omega)}^{p} \leq \lambda\left\|u_{-}\right\|_{L^{q}(\Omega)}^{p}\left|\Omega_{-}\right|^{1-p / q},
$$

which, by using that $u_{-} \not \equiv 0$ in $\Omega$, concludes the proof.

Remark 4.6. As an immediate consequence of Lemma 4.5, we have that if $\lambda \geq$ $\lambda_{1}(s, p)$, and $f \in W^{-s, p^{\prime}}(\Omega)$ is such that $f \leq 0$ and $f \not \equiv 0$, then there exist $\alpha>1$ and a constant $C>0$ such that for all $u$ is a weak sub-solution of (1.1) we have that

$$
\left(\frac{C}{\lambda}\right)^{\alpha} \leq\left|\Omega_{+}\right|
$$

where $\Omega_{+}=\{x \in \Omega: u(x)>0\}$.

Next, we prove our first anti-maximum principle.

Proof of Theorem 1.3. Again, we only prove the first statement; as before the another statement can be proved in an analogous way.

Suppose, to the contrary, there are sequences $\left\{\lambda_{n}\right\}_{n \in \mathbb{N}}$ and $\left\{u_{n}\right\}_{n \in \mathbb{N}}$ such that $\lambda_{n} \searrow \lambda_{1}(s, p)$ and $u_{n}$ is a weak solution of (1.1) with $\lambda=\lambda_{n}$ and $\left(u_{n}\right)_{+} \not \equiv 0$ for all $n \in \mathbb{N}$.

We claim that

$$
\left\|u_{n}\right\|_{L^{q}(\Omega)} \rightarrow \infty
$$

for all $p \leq q<p_{s}^{\star}$.

Suppose not, that is there is $q \in\left(p, p_{s}^{\star}\right)$ such that $\left\{u_{n}\right\}_{n \in \mathbb{N}}$ is bounded in $L^{q}(\Omega)$. Then, using that $u_{n}$ is a weak solution of (1.1) for all $n \in \mathbb{N}$, Hölder's inequality and $\lambda_{n} \searrow \lambda_{1}(s, p)$, we have that $\left\{u_{n}\right\}_{n \in \mathbb{N}}$ is bounded in $\widetilde{W}^{s, p}(\Omega)$. Then, since $T_{1}$ is a completely continuous operator (see Subsection 2.2), up to a subsequence (still denoted by $u_{n}$ )

$$
u_{n} \rightarrow u \text { strongly in } \widetilde{W}^{s, p}(\Omega),
$$

where $u$ is a weak solution of (1.1) with $\lambda=\lambda_{1}(s, p)$. By Corollary 4.3, this is a contradiction. We have prove our claim.

Set $q \in\left(p, p_{s}^{\star}\right)$ and

$$
v_{n}:=\frac{u_{n}}{\left\|u_{n}\right\|_{L^{q}(\Omega)}} \quad \forall n \in \mathbb{N} .
$$


Then for all $n \in \mathbb{N} v_{n}$ is a weak solution of

$$
\begin{cases}\left(-\Delta_{p}\right)^{s} u=\lambda_{n}|u|^{p-2} u+\frac{f(x)}{\left\|u_{n}\right\|_{L^{q}(\Omega)}^{p-1}} & \text { in } \Omega, \\ u=0 & \text { in } \Omega^{c} .\end{cases}
$$

Now, using again that $T_{1}$ is a completely continuous operator and the fractional Sobolev compact embedding theorem, up to a subsequence (still denoted by $v_{n}$ )

$$
\begin{array}{ll}
v_{n} \rightarrow v & \text { strongly in } \widetilde{W}^{s, p}(\Omega), \\
v_{n} \rightarrow v & \text { strongly in } L^{q}(\Omega) .
\end{array}
$$

Thus, $v \not \equiv 0$ in $\Omega$ and, $v$ is a weak solution of $(2.2)$ since $\lambda_{n} \rightarrow \lambda_{1}(s, p)$ and $f /\left\|u_{n}\right\|_{L^{q}(\Omega)}^{p-1} \rightarrow 0$ strongly in $W^{-s, p^{\prime}}(\Omega)$. That is $v$ is an eigenfunction of $\left(-\Delta_{p}\right)^{s}$ associated to $\lambda_{1}(s, p)$. Therefore either $v>0$ in $\Omega$ or $v<0$ in $\Omega$. The case $v>0$ is a contradiction by Lemma 4.5. To complete the proof of the theorem it remains to consider the case when $v<0$.

If $v<0$ then $\left(v_{n}\right)_{+} \rightarrow 0$ strongly in $L^{q}(\Omega)$. Therefore, using (4.1), it turns out that $\left\|\left(u_{n}\right)_{+}\right\|_{L^{q}(\Omega)} \rightarrow \infty$.

On the other hand, by the Sobolev embedding theorem, there is a constant $C$ independent of $n$ such that

$$
\begin{aligned}
C \mathcal{K} & \left\|\left(u_{n}\right)_{+}\right\|_{L^{q}(\Omega)}^{p} \leq \mathcal{K}\left|\left(u_{n}\right)_{+}\right|_{W^{s, p}\left(\mathbb{R}^{N}\right)}^{p} \\
& \leq \mathcal{K} \int_{\mathbb{R}^{2 N}} \frac{\left|u_{n}(x)-u_{n}(y)\right|^{p-2}\left(u_{n}(x)-u_{n}(y)\right)\left(\left(u_{n}\right)_{+}(x)-\left(u_{n}\right)_{+}(y)\right)}{|x-y|^{N+s p}} d x d y \\
& \leq \lambda_{n} \int_{\Omega}\left(u_{n}\right)_{+}^{p} d x+\left\langle f(x),\left(u_{n}\right)_{+}\right\rangle \\
& \leq \lambda_{n}\left\|\left(u_{n}\right)_{+}\right\|_{L^{q}(\Omega)}^{p}\left|\left\{x \in \Omega: u_{n}(x)>0\right\}\right|^{1-p / q}+\|f\|_{W^{-s, p^{\prime}}(\Omega)}\left|\left(u_{n}\right)_{+}\right|_{W^{s, p}\left(\mathbb{R}^{N}\right)}
\end{aligned}
$$

for all $n \in \mathbb{N}$. Then

$$
C \leq \lambda_{n}\left|\left\{x \in \Omega: u_{n}(x)>0\right\}\right|^{1-p / q}+\frac{\|f\|_{W^{-s, p \prime}(\Omega)}}{\left\|\left(u_{n}\right)_{+}\right\|_{L^{q}(\Omega)}^{p-1}}\left|v_{n}\right|_{W^{s, p}\left(\mathbb{R}^{N}\right)},
$$

for all $n \in \mathbb{N}$. Therefore

$$
\frac{C}{\lambda_{1}(s, p)} \leq \liminf _{n \rightarrow \infty}\left|\left\{x \in \Omega: u_{n}(x)>0\right\}\right|^{1-p / q},
$$

which is a contradiction with the fact that $\left(v_{n}\right)_{+} \rightarrow 0$ strongly in $L^{q}(\Omega)$.

Finally, We show our anti-maximum principle for the linear case.

Proof of Theorem 1.4. As before, we only prove the first statement; the other statements can be proved in an analogous way.

It is suffices to prove that, for any two sequences $\left\{\lambda_{n}\right\}_{n \in \mathbb{N}}$ and $\left\{u_{n}\right\}_{n \in \mathbb{N}}$ such that $\lambda_{n} \searrow \lambda_{1}(s, 2)$ and $u_{n}$ is a weak solution of (1.1) with $\lambda=\lambda_{n}$, there is $n_{0} \in \mathbb{N}$ such that $u_{n}<0$ in $\Omega$ for all $n \geq n_{0}$. For such sequences, by Lemma 2.6, $u_{n} \in L^{\infty}(\Omega)$ for all $n \in \mathbb{N}$.

We claim that

$$
\left\|u_{n}\right\|_{L^{\infty}(\Omega)} \rightarrow \infty
$$

Suppose not, that is $\{u\}_{n \in \mathbb{N}}$ is bounded in $L^{\infty}(\Omega)$. Then, using that $u_{n}$ is a weak solution of (1.1) for all $n \in \mathbb{N}$, Hölder's inequality and $\lambda_{n} \searrow \lambda_{1}(s, p)$, we have 
that $\left\{u_{n}\right\}_{n \in \mathbb{N}}$ is bounded in $\widetilde{W}^{s, 2}(\Omega)$. Then, since $T_{1}$ is a completely continuous operator, up to a subsequence (still denoted by $u_{n}$ )

$$
u_{n} \rightarrow u \text { strongly in } \widetilde{W}^{s, 2}(\Omega)
$$

where $u$ is a weak solution of (1.1) with $\lambda=\lambda_{1}(s, 2)$. Then

$$
\begin{aligned}
\lambda_{1}(s, 2) \int_{\Omega} u w_{1} d x & =\mathcal{K} \int_{\mathbb{R}^{2 k}} \frac{(u(x)-u(y))\left(w_{1}(x)-w_{1}(y)\right)}{|x-y|^{N+2 s}} d x d y \\
& =\lambda_{1}(s, 2) \int_{\Omega} u w_{1} d x+\int_{\Omega} f w_{1} d x .
\end{aligned}
$$

Therefore

$$
\int_{\Omega} f w_{1} d x=0
$$

and we have a contradiction. Thus our claim is proved.

Set

$$
v_{n}:=\frac{u_{n}}{\left\|u_{n}\right\|_{L^{\infty}(\Omega)}} \quad \forall n \in \mathbb{N}
$$

Then for all $n \in \mathbb{N} v_{n}$ is a weak solution of

$$
\begin{cases}\left(-\Delta_{p}\right)^{s} u=\lambda_{n}|u|^{p-2} u+\frac{f(x)}{\left\|u_{n}\right\|_{L^{\infty}(\Omega)}} & \text { in } \Omega, \\ u=0 & \text { in } \Omega^{c} .\end{cases}
$$

Now, using again that $T_{1}$ is a completely continuous operator and the fractional Sobolev compact embedding theorem, up to a subsequence (still denoted by $v_{n}$ )

$$
v_{n} \rightarrow v \quad \text { strongly in } \widetilde{W}^{s, 2}(\Omega)
$$

Thus, $v \not \equiv 0$ in $\Omega$ and, $v$ is a weak solution of $(2.2)$ since $\lambda_{n} \rightarrow \lambda_{1}(s, 2)$ and $f /\left\|u_{n}\right\|_{L^{\infty}(\Omega)} \rightarrow 0$ strongly in $\Omega$. That is $v$ is an eigenfunction of $(-\Delta)^{s}$ associated to $\lambda_{1}(s, 2)$. Therefore either $v>0$ in $\Omega$ or $v<0$ in $\Omega$.

On the other hand, for any $n \in \mathbb{N}$

$$
\left(\lambda_{1}(s, 2)-\lambda_{n}\right) \int_{\Omega} w_{1} v_{n} d x=\frac{1}{\|u\|_{L^{\infty}(\Omega)}} \int_{\Omega} f(x) w_{1} d x>0
$$

then, since $\lambda_{1}(s, 2)<\lambda_{n}$ for any $n \in \mathbb{N}$, we have that

$$
\int_{\Omega} w_{1} v_{n} d x<0 \quad \forall n \in \mathbb{N}
$$

Therefore $v<0$ in $\Omega$.

In addition, by Theorem 2.7 and the Arzela-Ascoli theorem, up to a subsequence (still denoted by $v_{n}$ )

$$
v_{n} \rightarrow w_{1} \quad \text { and } \quad \frac{v_{n}}{\delta^{s}} \rightarrow \frac{w_{1}}{\delta^{s}}
$$

strongly in $\bar{\Omega}$. Here $\delta(x)=\operatorname{dist}(x, \partial \Omega)$. Then, by Lemma 2.8 , there is $n_{0} \in \mathbb{N}$ such that $v_{n}<0$ for all $n \in \mathbb{N}$. That is there is $n_{0} \in \mathbb{N}$ such that $u_{n}<0$ for all $n \geq n_{0}$.

Aknowledgements. L. M. Del Pezzo was partially supported by CONICET PIP 5478/1438 (Argentina) and A. Quaas was partially supported by Fondecyt grant No. 1110210, Millennium Nucleus Center for Analysis of PDE NC130017 and Basal CMM UChile. 


\section{REFERENCES}

[1] R. A. Adams, Sobolev spaces, Academic Press [A subsidiary of Harcourt Brace Jovanovich, Publishers], New York-London, 1975, Pure and Applied Mathematics, Vol. 65. MR 0450957 (56 \#9247)

[2] S. Amghibech, On the discrete version of Picone's identity, Discrete Appl. Math. 156 (2008), no. 1, 1-10. MR 2363452 (2008m:35090)

[3] A. Anane, Simplicité et isolation de la première valeur propre du p-laplacien avec poids, C. R. Acad. Sci. Paris Sér. I Math. 305 (1987), no. 16, 725-728. MR 920052 (89e:35124)

[4] A. Anane, and J. Gossez, Strongly nonlinear elliptic problems near resonance: a variational approach, Comm. Partial Differential Equations 15 (1990), no. 8, 1141-1159.

[5] A. Anane and N. Tsouli, On a nonresonance condition between the first and the second eigenvalues for the p-Laplacian, Int. J. Math. Math. Sci. 26 (2001), no. 10, 625-634. MR 1861112

[6] - On a nonresonance condition between the first and the second eigenvalue for the p-Laplacian, Math-Rech. Appl. 6 (2004), 101-114. MR 2604105

[7] D. Arcoya, E. Colorado, and T. Leonori, Asymptotically linear problems and antimaximum principle for the square root of the Laplacian, Adv. Nonlinear Stud. 12 (2012), no. 4, 683-701. MR 3012856

[8] D. Arcoya and J. L. Gámez, Bifurcation theory and related problems: anti-maximum principle and resonance, Comm. Partial Differential Equations 26 (2001), no. 9-10, 1879-1911. MR 1865948

[9] D. Arcoya and L. Orsina, Landesman-Lazer conditions and quasilinear elliptic equations, Nonlinear Anal. 28 (1997), no. 10, 1623-1632. MR 1430505

[10] M. Arias, J. Campos, and J.-P. Gossez, On the antimaximum principle and the Fučik spectrum for the Neumann p-Laplacian, Differential Integral Equations 13 (2000), no. 1-3, 217226. MR 1811956

[11] S. N. Armstrong, Principal eigenvalues and an anti-maximum principle for homogeneous fully nonlinear elliptic equations, J. Differential Equations 246 (2009), no. 7, 2958-2987. MR 2503031

[12] I. Birindelli, Hopf's lemma and anti-maximum principle in general domains, J. Differential Equations 119 (1995), no. 2, 450-472. MR 1340547

[13] L. Boccardo, P. Drábek, D. Giachetti, and M. Kučera, Generalization of Fredholm alternative for nonlinear differential operators. Nonlinear Anal., 10 (1986), 1083-1103.

[14] J. Bourgain, H. Brezis, and P. Mironescu, Another look at sobolev spaces, in: Optimal Control and Partial Differential Equations, 2001, 439-455.

[15] L. Brasco and G. Franzina, Convexity properties of Dirichlet integrals and Picone-type inequalities, Kodai Math. J. 37 (2014), no. 3, 769-799. MR 3273896

[16] L. Brasco and E. Parini, The second eigenvalue of the fractional p-laplacian, Adv. Calc. Var. 9 (2016), no. 4, 323-355.

[17] L. Brasco, E. Parini, and M. Squassina, Stability of variational eigenvalues for the fractional p-Laplacian, Discrete Contin. Dyn. Syst. 36 (2016), no. 4, 1813-1845. MR 3411543

[18] Ph. Clément and L. A. Peletier, An anti-maximum principle for second-order elliptic operators, J. Differential Equations 34 (1979), no. 2, 218-229. MR 550042

[19] M. Cuesta, D. de Figueiredo, and J.-P. Gossez, The beginning of the Fučik spectrum for the p-Laplacian, J. Differential Equations 159 (1999), no. 1, 212-238. MR 1726923

[20] L. M. Del Pezzo, J. Fernández Bonder and L. López Ros, An optimization problem for the first eigenvalue of the p-fractional Laplacian, arXiv:1601.03019.

[21] L. M. Del Pezzo and A. Quaas, Global bifurcation for fractional p-Laplacian and application, Z. Anal. Anwend. 35 (2016), no. 4, 411447.

[22] L. M. Del Pezzo and A. Quaas, A Hopf's lemma and a strong minimum principle for the fractional p-Laplacian, arXiv:1609.04725

[23] M. del Pino, P. Drábek, and R. Manásevich, The Fredholm alternative at the first eigenvalue for the one-dimensional p-Laplacian, J. Differential Equations 151 (1999), no. 2, 386-419. MR 1669705

[24] K. Deimling, Nonlinear Functional Analysis, Springer-Verlag, Berlin, 1985. xiv+450 pp.

[25] F. Demengel and G. Demengel, Functional spaces for the theory of elliptic partial differential equations, Universitext, Springer, London, 2012, Translated from the 2007 French original by Reinie Erné. MR 2895178 
[26] E. Di Nezza, G. Palatucci, and E. Valdinoci, Hitchhiker's guide to the fractional Sobolev spaces, Bull. Sci. Math. 136 (2012), no. 5, 521-573. MR 2944369

[27] C. L. Dolph, Nonlinear integral equations of the Hammerstein type, Trans. Amer. Math. Soc. 66 (1949), 289-307. MR 0032923

[28] P. Drábek, The p-Laplacian-mascot of nonlinear analysis, Acta Math. Univ. Comenian. (N.S.) 76 (2007), no. 1, 85-98. MR 2331056

[29] P. Drábek, P. Girg, P. Takáč, and M. Ulm, The Fredholm alternative for the p-Laplacian: bifurcation from infinity, existence and multiplicity, Indiana Univ. Math. J. 53 (2004), no. 2, 433-482. MR 2060041 (2005f:35095)

[30] P. Drábek, P. Girg, and R. Manásevich, Generic Fredholm alternative-type results for the one dimensional p-Laplacian, NoDEA Nonlinear Differential Equations Appl. 8 (2001), no. 3, 285-298. MR 1841260

[31] P. Drábek and P. Takáč, A counterexample to the Fredholm alternative for the p-Laplacian, Proc. Amer. Math. Soc. 127 (1999), no. 4, 1079-1087. MR 1646309

[32] J. Fleckinger, J.-P. Gossez, P. Takáč, and François de Thélin, Existence, nonexistence et principe de l'antimaximum pour le p-laplacien, C. R. Acad. Sci. Paris Sér. I Math. 321 (1995), no. 6, 731-734. MR 1354715

[33] G. Franzina and G. Palatucci, Fractional p-eigenvalues, Riv. Math. Univ. Parma (N.S.) 5 (2014), no. 2, 373-386. MR 3307955

[34] J. García-Melián and J. D. Rossi, Maximum and antimaximum principles for some nonlocal diffusion operators, Nonlinear Anal. 71 (2009), no. 12, 6116-6121. MR 2566516

[35] T. Godoy, J.-P. Gossez, and S. Paczka, On the antimaximum principle for the p-Laplacian with indefinite weight, Nonlinear Anal. 51 (2002), no. 3, 449-467. MR 1942756

[36] J.-P. Gossez, Sur le principe de l'antimaximum, Cahiers Centre Études Rech. Opér. 36 (1994), 183-187, Hommage à Simone Huyberechts. MR 1376860

[37] A. Greco and R. Servadei, Hopf's lemma and constrained radial symmetry for the fractional Laplacian. (English summary) Math. Res. Lett. 23 (2016), no. 3, 863-885. MR 3533199

[38] P. Grisvard, Elliptic problems in nonsmooth domains, Monographs and Studies in Mathematics, vol. 24, Pitman (Advanced Publishing Program), Boston, MA, 1985. MR 775683 (86m:35044)

[39] A. Iannizzotto, S. Mosconi, and M. Squassina, Global Hölder regularity for the fractional $p$-Laplacian, To appear in Revista Matemática Iberoamericana (2014).

[40] E. Lindgren and P. Lindqvist, Fractional eigenvalues, Calc. Var. Partial Differential Equations 49 (2014), no. 1-2, 795-826. MR 3148135

[41] P. Lindqvist, On the equation $\operatorname{div}\left(|\nabla u|^{p-2} \nabla u\right)+\lambda|u|^{p-2} u=0$, Proc. Amer. Math. Soc. 109 (1990), no. 1, 157-164. MR 1007505

[42] Addendum: "On the equation $\operatorname{div}\left(|\nabla u|^{p-2} \nabla u\right)+\lambda|u|^{p-2} u=0$ ", Proc. Amer. Math. Soc. 116 (1992), no. 2, 583-584. MR 1139483

[43] R. Manásevich and P. Takáč, On the Fredholm alternative for the p-Laplacian in one dimension, Proc. London Math. Soc. (3) 84 (2002), no. 2, 324-342. MR 1881394

[44] E. Parini, Continuity of the variational eigenvalues of the p-Laplacian with respect to $p$, Bull. Aust. Math. Soc. 83 (2011), no. 3, 376-381. MR 2794523

[45] X. Ros-Oton and J. Serra, The Dirichlet problem for the fractional Laplacian: regularity up to the boundary, J. Math. Pures Appl. (9) 101 (2014), no. 3, 275-302. MR 3168912

[46] R. Servadei and E. Valdinoci, Variational methods for non-local operators of elliptic type, Discrete Contin. Dyn. Syst. 33 (2013), no. 5, 2105-2137. MR 3002745

[47] _ A Brezis-Nirenberg result for non-local critical equations in low dimension. Commun. Pure Appl. Anal. 12 (2013), no. 6, 2445-2464. MR MR3060890

[48] On the spectrum of two different fractional operators, Proc. Roy. Soc. Edinburgh Sect. A 144 (2014), no. 4, 831-855. MR 3233760

[49] — Weak and viscosity solutions of the fractional Laplace equation, Publ. Mat. 58 (2014), no. 1, 133-154. MR 3161511

[50] P. Takáč, On the Fredholm alternative for the p-Laplacian at the first eigenvalue, Indiana Univ. Math. J. 51 (2002), no. 1, 187-237. MR 1896161

[51] A variational approach to the Fredholm alternative for the $p$-Laplacian near the first eigenvalue, J. Dynam. Differential Equations 18 (2006), no. 3, 693-765. MR 2264042 
L. M. Del Pezzo

Conicet and Departamento de Matemática, FCEyn, Universidad de Buenos Aires,

Pabellon I, Ciudad Universitaria (1428), Buenos Aires, Argentina.

E-mail address: Idpezzo@dm.uba.ar

A. QuAas

Departamento de Matemática, Universidad técnica Federico Santa María Casilla V-110, Avda. España, 1680 - Valparaíso, CHILE.

E-mail address: alexander.quaas@usm.cl 